\title{
Lock-and-Key Exciplexes for Thermally Activated Delayed Fluorescence
}

\author{
Constantin-Christian A. Voll ${ }^{a}$ \\ Georgios Markopoulos ${ }^{a}$ \\ Tony C. Wu ${ }^{\mathbf{b}}$ \\ Matthew Welborn ${ }^{a}$ \\ Jens U. Engelhart ${ }^{a}$ \\ Sébastien Rochat ${ }^{a}$ \\ Grace G. D. Han ${ }^{a}$ \\ Graham T. Sazama ${ }^{a}$ \\ Ting-An Lin ${ }^{b}$ \\ Troy Van Voorhis ${ }^{a}$ \\ Marc A. Baldo*b \\ Timothy M. Swager ${ }^{* a}$ (D) \\ Department of Chemistry, Massachusetts Institute of Technology, Cambridge, \\ Massachusetts, United States \\ tswager@mit.edu \\ b Department of Electrical Engineering and Computer Science, Massachusetts \\ Institute of Technology, Cambridge, Massachusetts, United States
}

Received: 19.10 .2019

Accepted after revision: 05.11.2019

DOI: 10.1055/s-0039-3402059; Art ID: om-19-0020-oa

License terms: (c)

(c) 2020. The Author(s). This is an open access article published by Thieme under the terms of the Creative Commons Attribution-NonDerivative-NonCommercial-License, permitting copying and reproduction so long as the original work is given appropriate credit. Contents may not be used for commercial purposes, or adapted, remixed, transformed or built upon. (https://creativecommons.org/licenses/by-nc-nd/4.0/)

Abstract We combine synthetic supramolecular chemistry and materials science to develop novel exciplexes for thermally activated delayed fluorescence. Our approach starts from a bowl-shaped acceptor molecule for which we synthesize tailor-made donors that bind in a lockand-key fashion. The donor design is guided by extensive density functional theory calculations of three independent donor families. The investigation of a large number of custom-synthesized donors allows us to derive empirical relationships for the prediction of the exciplex emission color. Incorporated within organic light-emitting devices, the lock-and-key exciplexes yield external quantum efficiencies of up to $5.4 \%$, with potentially tunable emission color across the blue and green visible spectrum.

Key words TADF, exciplex, OLED, supramolecular chemistry, emitter

\section{Introduction}

The advent of thermally activated delayed fluorescence (TADF) has led to a revolution in the field of organic lightemitting diodes (OLEDs). ${ }^{1-5}$ By designing compounds with small singlet-triplet gaps, the triplet excitons formed during OLED operation can be harvested through thermally revers-

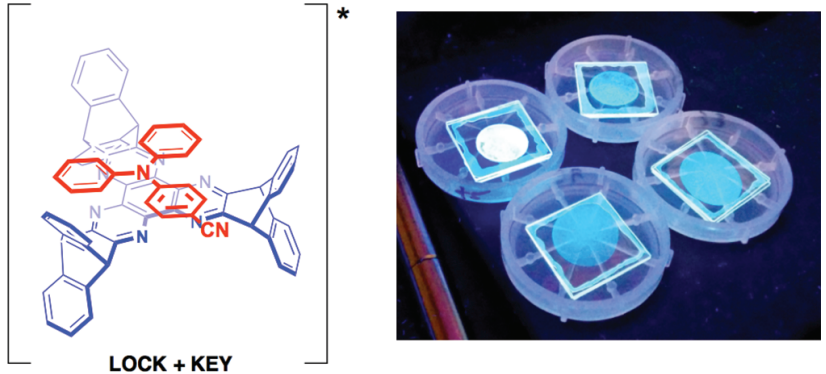

ible intersystem crossing. External quantum efficiencies (EQEs) of 30\% have been reported for OLEDs that include intramolecular TADF emitters. ${ }^{6-9}$ These small singlet-triplet gaps can be achieved through spatial separation of donor and acceptor moieties, either through a twisted donor-acceptor approach $^{10}$ or homoconjugation (Figure 1). ${ }^{11-13}$ Either approach spatially separates the HOMO and LUMO orbitals, which results in a decreased exchange energy and small singlet-triplet gaps. The search for new TADF materials has furthermore revived an interest in exciplexes as emissive materials (Figure 1). ${ }^{14-37}$ Since the HOMO and LUMO orbitals of an exciplex are located on entirely different molecules, exciplexes intrinsically have a small singlet-triplet gap. Exciplex-based OLEDs have achieved remarkable EQEs, ${ }^{38}$ either as pure exciplex emitters or when formed with an intrinsic TADF emitter, with some devices exceeding 20\%. ${ }^{39,40}$

In addition to interfacial challenges, ${ }^{41}$ low fluorescence quantum yields limit exciplex-based device efficiency. The excited complex between two molecules is weakly bonded and its flat, shallow bonding potential gives rise to nonradiative decay channels. These limitations impose the need for an extensive structure and device optimization to achieve high efficiencies.

In order to address this limitation of exciplexes, we draw upon a central concept of supramolecular chemistry: more than 100 years ago Emil Fischer introduced the lock-and-key hypothesis to describe the action of enzymes on their substrates. ${ }^{42,43}$ Enzymes can only work on substrates for which a geometrical fit is possible. Exciplexes are supramolecular entities and, consequently, geometrical complementarity will also play a role in determining their physical properties. The influence of geometrical considerations has occasionally 


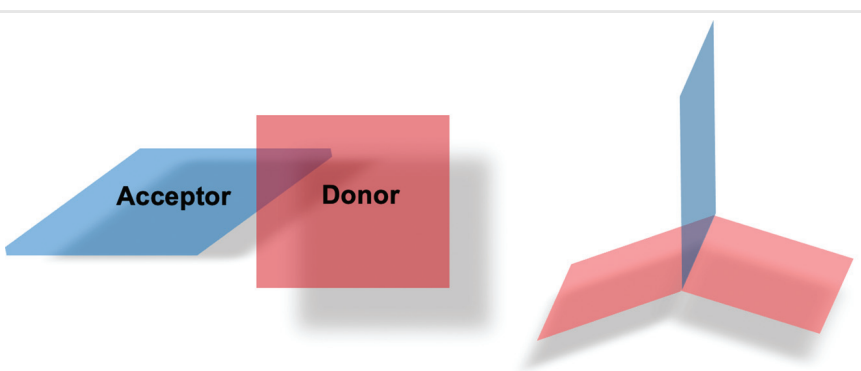

Twisted Donor-Acceptor
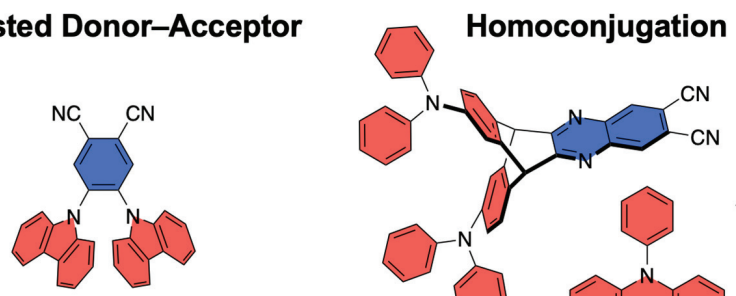

Homoconjugation

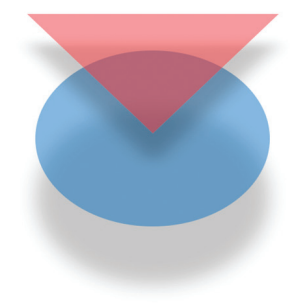

Exciplex

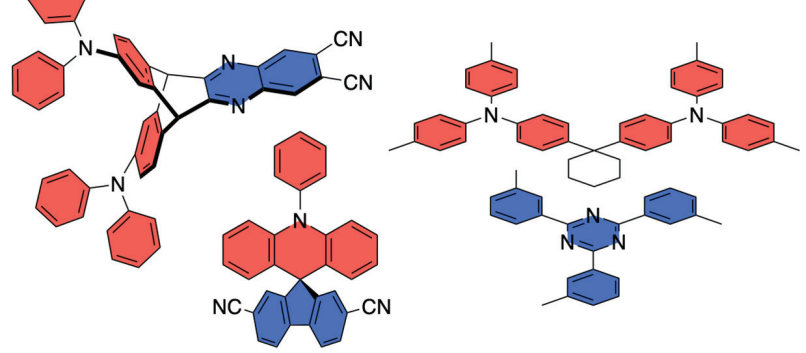

Figure 1 Overview of classes of TADF emitters. Molecules featuring a large torsional angle between the donor and acceptor (left), homoconjugated molecules (middle), and exciplexes (right).

been mentioned in the literature on TADF exciplexes, ${ }^{26,32}$ but no systematic study on these parameters has been performed. In the present work, we propose a lock-and-key approach to exciplex design wherein an acceptor and a donor are tailormade to fit each other. The molecular recognition in the ground state leads to a preorganization of the exciplex geometry, giving rise to a more rigid and consequently more emissive exciplex upon excitation.

For our molecular design we envisioned a concave binding pocket that binds the other component through a close fit. Such a binding pocket is realized in compound $\mathbf{1}$ (Figure 2 ) where the electron-accepting hexaazatriphenylene core is flanked by three triptycene moieties. Hexaazatriphenylene derivatives have previously been used as OLED materials. ${ }^{44-53}$ Triptycenes, on the other hand, have been shown to be very effective in

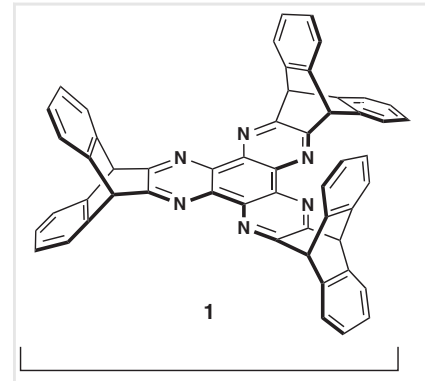

"LOCK"

acceptor with binding pocket

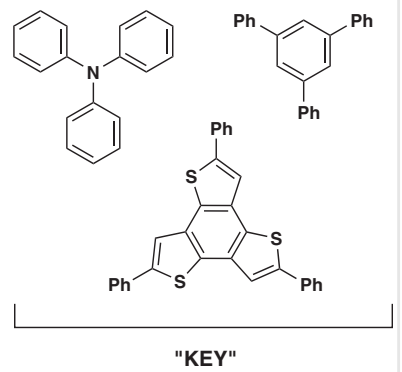

3 shape-selected donor families
Figure 2 Tailor-made acceptor and shape-selected donors in this work. introducing free volume that can be used for molecular recognition processes. ${ }^{54}$ Because of the $C_{3}$-symmetric nature of the acceptor $\mathbf{1}$, we explored donor frameworks that reflect this symmetry. Three distinct donor families were chosen for geometrical complementarity: triarylamines, triarylbenzenes, and triarylbenzotrithiophenes (Figure 2), and their exciplex properties with acceptor $\mathbf{1}$ were studied.

\section{Results and Discussion}

\section{Acceptor Synthesis and Properties}

The acceptor (1) was prepared on a gram scale in 55\% yield through condensation of hexaaminobenzene trihydrochloride $^{55}$ (2) with diketone $\mathbf{3}^{56}$ (Scheme 1). The colorless solid has a generally low solubility, with chloroform being the best among the commonly used solvents. Crystals were grown by slow diffusion of pentane into a dichloromethane solution of $\mathbf{1}$. As a result of the large proportion of disordered solvent molecules in the crystal, X-ray crystallographic data of quality sufficient for publication could not be obtained. However, the general connectivity and crystal packing information regarding acceptor $\mathbf{1}$ could be determined (see the Supporting Information). We observed that the molecules stacked in dimers that were mediated by $\pi / \pi$-interactions between two triptycene wings. The large void spaces created by the remaining triptycene wings are filled with heavily disordered dichloromethane and pentane molecules. 


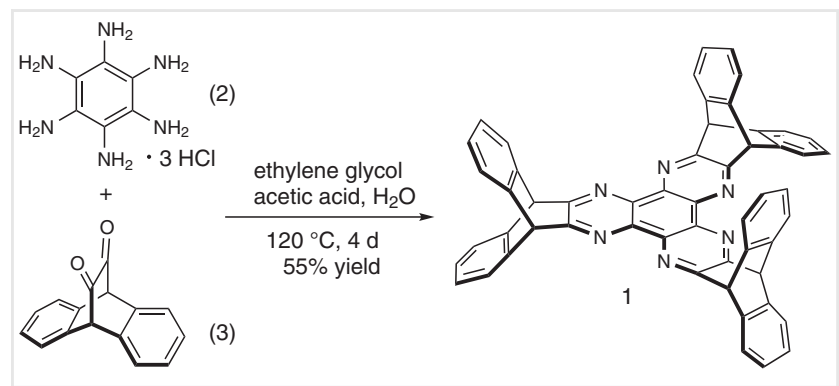

Scheme 1 Synthesis of the acceptor.

Acceptor 1 has absorption maxima at 336 and $356 \mathrm{~nm}$ (Figure 3). While the absorption spectra are essentially the same in solution and thin films, marked differences were observed for the photoluminescence (PL) spectra. In solution the compound emits at $383 \mathrm{~nm}$ with a shoulder at $363 \mathrm{~nm}$. In contrast, the thin-film emission exhibits three maxima at 392, 420, and $442 \mathrm{~nm}$. We hypothesized that the peak at $392 \mathrm{~nm}$ corresponds to unperturbed 1 similar to that observed in solution with a slight red-shift attributed to solid-state effects. To gain better understanding of the broad features at 420 and $442 \mathrm{~nm}$, we measured the PL of acceptor 1 in a poly(methyl methacrylate) (PMMA) matrix. The resulting PL spectrum had a single maximum at $383 \mathrm{~nm}$ similar to 1's solution PL. This finding indicated that the thin-film peaks at 420 and $442 \mathrm{~nm}$ arise from aggregationinduced effects. This is further supported through the observation that the intensity ratios of the three maxima in the thin film vary between different spin-coated samples.

The electronic structure of acceptor $\mathbf{1}$ was further elucidated by cyclic voltammetry (Table 1 ). The compound exhibited a reversible redox behavior in dichloromethane solution, and a single redox peak was observed at $-1.90 \mathrm{~V}$ (vs. $\mathrm{Ag} / \mathrm{Ag}^{+}$). Compared to parent hexaazatriphenylene ${ }^{57,58}$

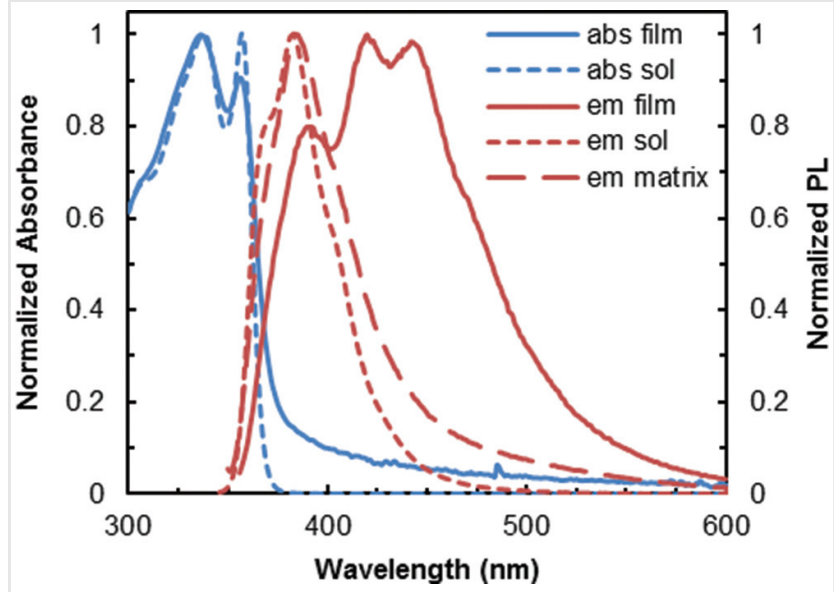

Figure 3 Absorption and photoluminescence (PL) spectra of acceptor 1 in thin film, solution, and PMMA matrix (abs = absorption, em = emission, sol $=$ solution).
Table 1 HOMO and LUMO energies of acceptor 1 derived from cyclic voltammetry and UV-vis absorption

\begin{tabular}{|c|c|c|c|c|c|}
\hline $\begin{array}{l}E_{\text {redox }} \\
{[\mathrm{V}]^{\mathrm{a}}}\end{array}$ & $\begin{array}{l}E_{\text {onset }} \\
{[\mathrm{V}]^{\mathrm{b}}}\end{array}$ & $\mathrm{V}]^{\mathrm{C}}$ & $\begin{array}{l}\lambda_{\text {onset }} \\
{[\mathrm{nm}]^{\mathrm{d}}}\end{array}$ & $\begin{array}{l}E_{\text {gap }} \\
{[\mathrm{eV}]^{\mathrm{e}}}\end{array}$ & $\begin{array}{l}\mathrm{HOMO} \\
{[\mathrm{eV}]^{\mathrm{f}}}\end{array}$ \\
\hline 1.90 & -1.80 & & 0 & 3.3 & -6.69 \\
\hline \multicolumn{6}{|c|}{ 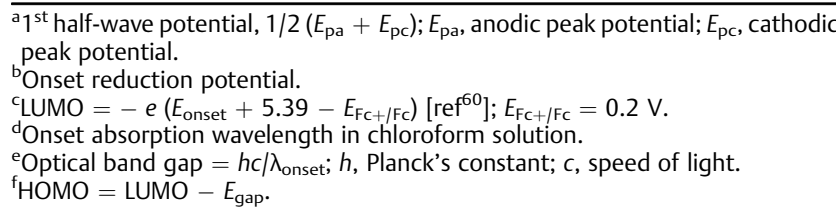 } \\
\hline
\end{tabular}

$\left(E_{1 / 2}=-1.44 \mathrm{eV}\right)$, the reduction potential of acceptor $\mathbf{1}$ is shifted to more negative potentials. This indicates a more electron-rich character of $\mathbf{1}$ that can be explained by electron donation via homoconjugation from the triptycene wings. ${ }^{59}$ The LUMO level $(-3.39 \mathrm{eV})$ was subsequently calculated from the onset reduction potential of the compound relative to the internal ferrocene/ferrocenium standard potential. The optical band gap estimated from the onset absorption wavelength was then subtracted from the LUMO level to calculate a HOMO level of $-6.69 \mathrm{eV}$.

\section{Exciplex Studies}

Exciplex formation was studied with 1:1 mixtures of donors and acceptors. Measurements were performed on spincoated thin films. No exciplexes were observed in solution as a result of the limited solubility of acceptor $\mathbf{1}$ that prevented the concentrations necessary for exciplex formation.

The thin-film PL spectra of all donors and their 1:1 mixture with acceptor 1 are shown in the Supporting Information. We discuss the general features of these spectra with triphenylamine (X01) as an exemplary donor (Figure 4). The PL of

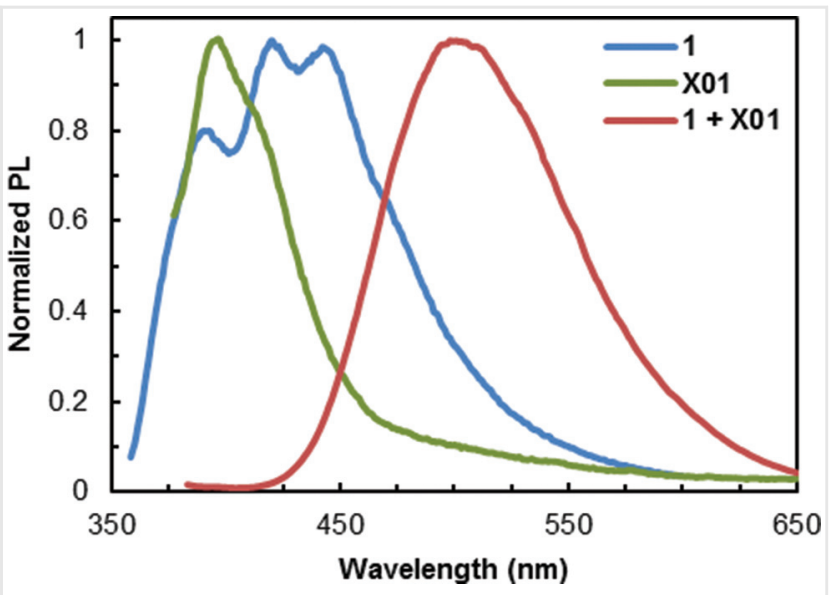

Figure 4 Fluorescence spectra of acceptor $\mathbf{1}$, donor $\mathbf{X 0 1}$, and their 1:1 mixture. 
triphenylamine has a maximum at $396 \mathrm{~nm}$; the PL of acceptor 1 with its peaks at 392, 420, and $442 \mathrm{~nm}$ has been described above. The PL of a 1:1 mixture of 1 and X01 exhibited a single maximum at $500 \mathrm{~nm}$. The pronounced red-shift compared to the individual components is typical for an exciplex. Only the exciplex emission is visible in the mixture; the contributions of the individual components are no longer visible. To exclude the action of a solvatochromic effect, we performed ratio experiments in which the acceptor was gradually added to the donor, and vice versa (see the Supporting Information). We unambiguously observed that the exciplex peak develops independently of the acceptor and donor peaks.

\section{Triarylamine Donors}

Molecular modeling indicated that the $C_{3}$-symmetrical core of triarylamines possesses a good geometrical fit to the cavity of the acceptor (Figure 5b). We first explored exciplex formation with a series of commercially available triarylamines (Figure 5a). In addition to the triarylamines X01-X03, we also included X04 (TPD) and X05 (TCTA) that are wellknown hole-transporting materials in OLED devices. ${ }^{61}$ For the benzidine TPD (X04), we hypothesized that only one of the triarylamine units would bind to the acceptor. The exciplexes of compounds X01-X05 with acceptor $\mathbf{1}$ emitted in the range of 500-537 $\mathrm{nm}$. Because of the general interest in blue OLED materials, we focused on synthesizing electron-poorer triarylamines to achieve a blue-shift of the emission (for an explanation of this rationale, see the section "Color Tuning of Exciplex Emission"). Substituted triarylamines were prepared by a $\mathrm{C}-\mathrm{N}$ coupling methodology ${ }^{62}$ and initial targets included cyano, trifluoromethyl, and fluorine substitution (compounds B01-B06 in Figure 5c). The exciplex emission was, as expected, blue-shifted to 442-496 nm. Qualitative comparison of the exciplexes indicated that cyano-substituted donors led to brighter exciplexes. Therefore, the cyano motif was further explored by synthesizing B09-B11 (Figure 5c). Compound B09 constitutes a heavier analogue of B01 and improves practicability in the thermal evaporation step for OLED fabrication. The benzidines B10 and B11 were inspired by the TPD motif (X04 in Figure 5a). The respective exciplexes with acceptor $\mathbf{1}$ emitted in the range of 473-507 nm.

Dispersion-corrected density functional theory was employed to calculate the binding energies between the donor and acceptor in the ground state. For the triarylamine family, binding energies of $25-46 \mathrm{kcal} / \mathrm{mol}$ were obtained. The parent triarylamine $\mathbf{X 0 1}$ is at the low end of the scale, whereas triarylamine B09 exhibits the largest binding energy. These values compare nicely with the calculated binding energies in molecular tweezers that are known to be effective hosts for
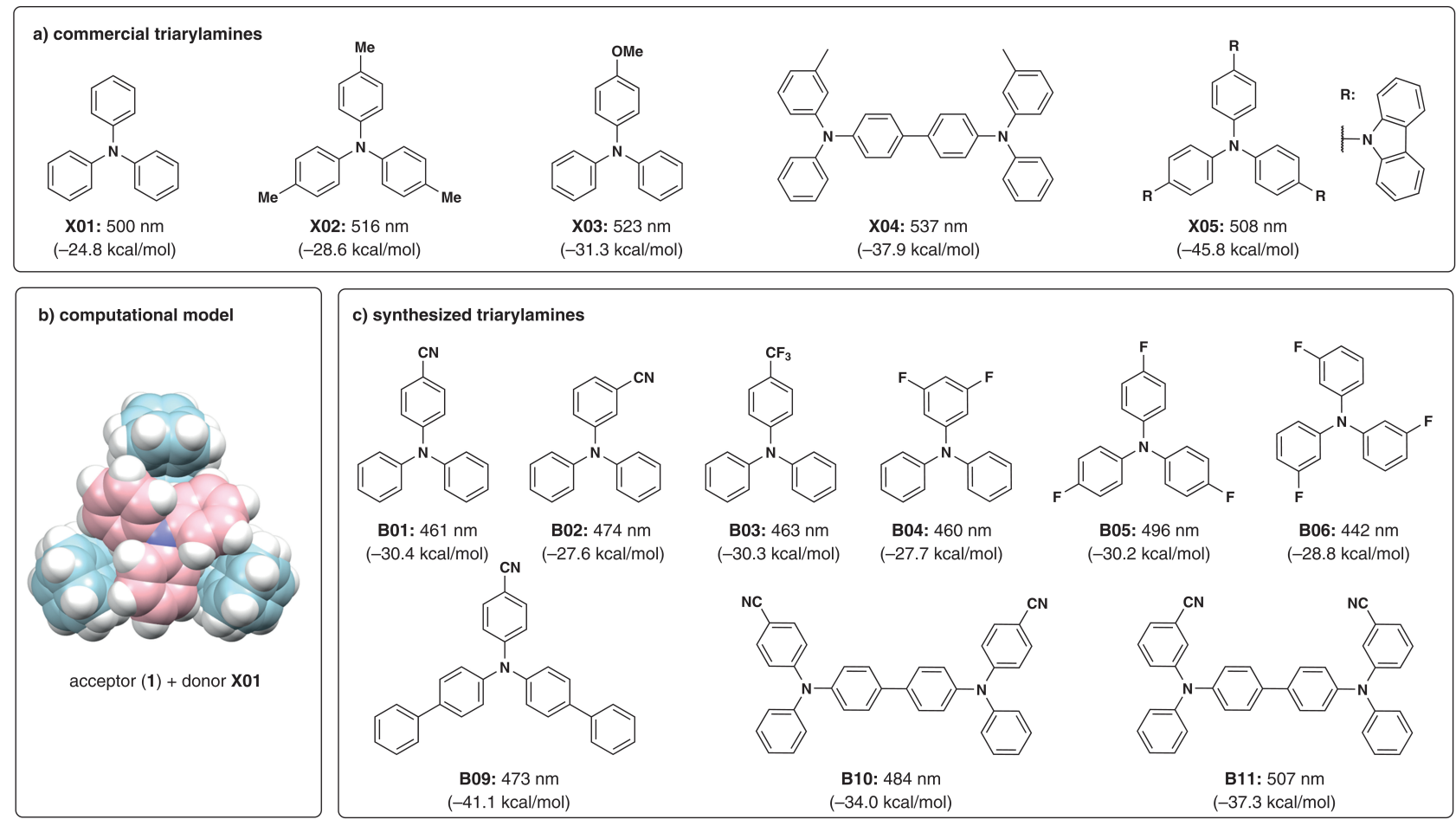

Figure 5 Triarylamine donors and their exciplex properties with acceptor 1 (exciplex emission wavelength and calculated binding energy at B3LYP-D/aug-cc-pVDZ//B3LYP-D/6-31 + G*). 
aromatic substrates. ${ }^{63}$ Closer inspection of the calculated geometries revealed that only $C_{3}$-symmetric donors and $\mathbf{B 0 2}$ are perfectly poised at the center of the acceptor cavity. Non$C_{3}$-symmetrical donors except $\mathbf{B 0 2}$ are either displaced towards a triptycene wing (X03, X04, B01, B03, B10, B11) or towards the void between two triptycene wings (B04, B09).

The large library of triarylamine donors with minute electronic differences allowed us to exhaustively cover a broad range of emission wavelengths. For example, in Figure 6 we plot the PL of seven selected exciplexes that cover the emission range from 461 to $537 \mathrm{~nm}$. In fact, the growing data set in the course of this investigation allowed us to derive empirical relationships that guided the synthesis of donors with exciplex emission wavelengths that were not yet covered by our molecules. These relationships are discussed in detail in the section "Color Tuning of Exciplex Emission."

\section{Triarylbenzene Donors}

We next turned our attention to $C_{3}$-symmetrical triarylbenzenes because computational modeling demonstrated their fit into the cavity of acceptor 1 (Figure 7). Compounds in this family were prepared by a standard Suzuki crosscoupling methodology. The PL maximum of a 1:1 mixture of donor $\mathbf{A 0 1}$ with acceptor $\mathbf{1}$ was observed at $431 \mathrm{~nm}$; this is a minimal redshift compared to the pure acceptor emission, and therefore the formation of an exciplex had to be questioned. In order to red-shift the emission into the visible region and away from the acceptor emission, we synthesized the more electron-rich derivatives $\mathbf{A 0 2}$ and $\mathbf{A 0 3}$ (Figure 7). The design idea behind $\mathbf{A 0 3}$ was that the dioxymethylene bridges are more rigid than the methoxy groups in $\mathbf{A 0 2}$ and that increased rigidity will favor radiative emission over other decay channels. The resulting exciplexes indeed exhibited a red-shift with emissions at 458 and $448 \mathrm{~nm}$, respectively (see

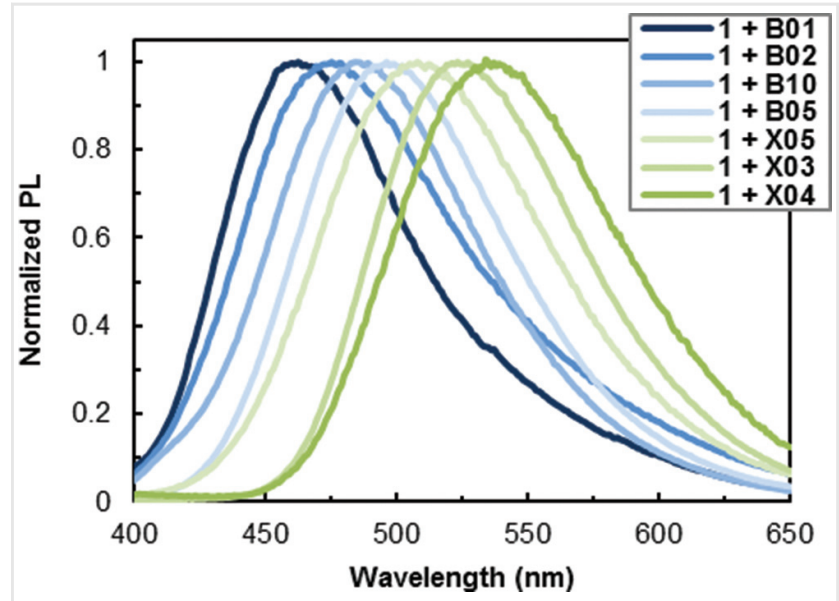

Figure 6 Tunability of exciplex emission through variation of triarylamine donor.
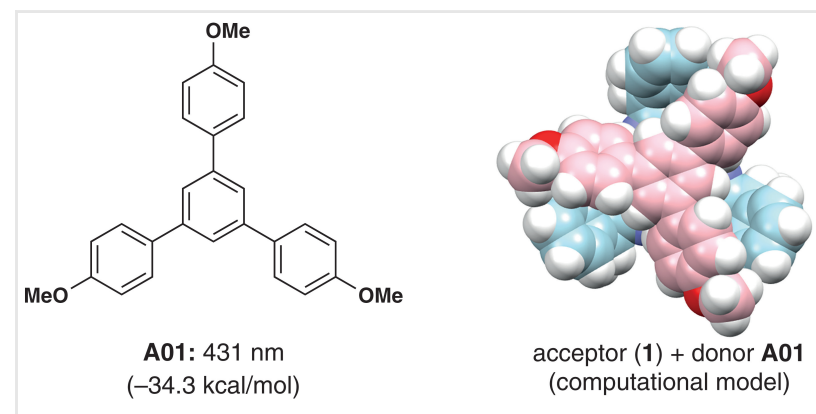

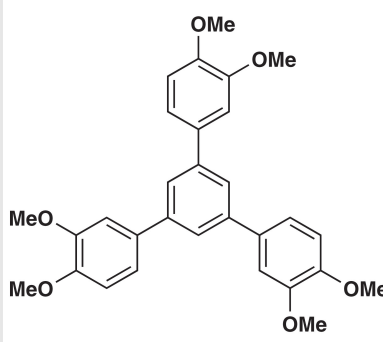

A02: $458 \mathrm{~nm}$

$(-38.8 \mathrm{kcal} / \mathrm{mol})$

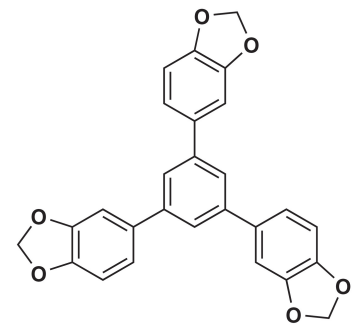

A03: $448 \mathrm{~nm}$

$(-36.5 \mathrm{kcal} / \mathrm{mol})$
Figure 7 Triarylbenzene donors and their exciplex properties with acceptor 1 (exciplex emission wavelength and calculated binding energy at B3LYP-D/aug-cc-pVDZ//B3LYP-D/6-31 + $\mathrm{G}^{*}$ ).

the Supporting Information). These emission bands are clearly separated from the potential acceptor emission, so that efficient exciplex formation can be assumed. Again, density functional theory was employed to assess binding energies. The calculated binding energies for the ground state complexes of A01-A03 with acceptor $\mathbf{1}$ range from 34 to 39 $\mathrm{kcal} / \mathrm{mol}$, which is comparable to the values for the triarylamine donor family above. Donor A02 has the highest binding energy of the triarylbenzene donors.

\section{Benzotrithiophene Donors}

Another promising $C_{3}$-symmetrical motif for donors is the benzotrithiophene framework (Figure 8). We prepared the triphenyl derivative $\mathbf{C O 1}^{64}$ and studied its exciplex properties with acceptor 1 . A weak emission at $453 \mathrm{~nm}$ was observed that is in the range of both acceptor and donor emission. As a result, no clear evidence for an exciplex could be observed in this case despite the excellent geometric fit. The calculated binding energy of the ground state complex amounts to $38.4 \mathrm{kcal} / \mathrm{mol}$.

\section{Color Tuning of Exciplex Emission}

We have shown that the emission wavelength of the exciplexes can be adjusted by synthesizing donors with 


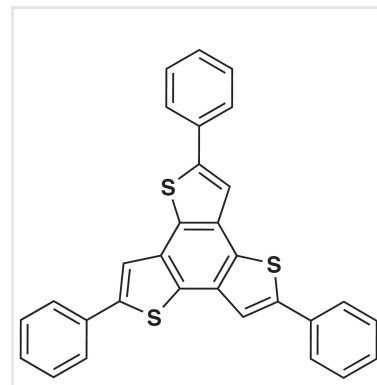

C01: $453 \mathrm{~nm}$ $(-38.4 \mathrm{kcal} / \mathrm{mol})$

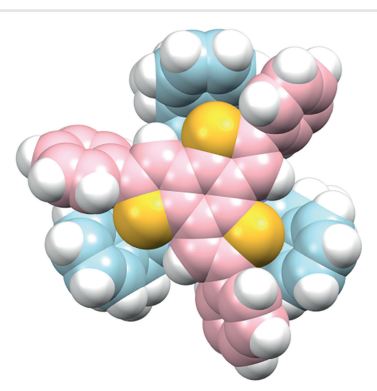

acceptor (1) + donor C01 (computational model)
Figure 8 Triphenylbenzotrithiophene and its exciplex properties with acceptor 1 (exciplex emission wavelength and calculated binding energy at B3LYP-D/aug-cC-pVDZ//B3LYP-D/6-31 + $\mathrm{G}^{*}$ ).

electron-donating or -withdrawing groups. It is well known that exciplexes display a linear relationship between the exciplex emission energy and the difference between the electron affinity (EA) of the acceptor and the ionization potential (IP) of the donor: $h \lambda_{\text {exciplex }}=\left(E_{\text {acceptor }}-\mathrm{IP}_{\text {donor }}\right)+$ const. $^{65,66}$ To study this relationship we calculated the EA of acceptor $1(0.97 \mathrm{eV})$ and the IP of the various triarylamine donors X01-B11 (see the Supporting Information). In Figure 9 we plot the experimental exciplex emission wavelengths against the calculated EA - IP differences for the exciplexes of 1 with triarylamines X01-B11. ${ }^{67,68}$ The resulting empirical relationship can be used to design donors with a specific exciplex emission wavelength. For example, for blue exciplexes (450-480 nm) one should synthesize a triarylamine that yields a theoretical EA - IP difference of 5.8-6.3 eV. Since the

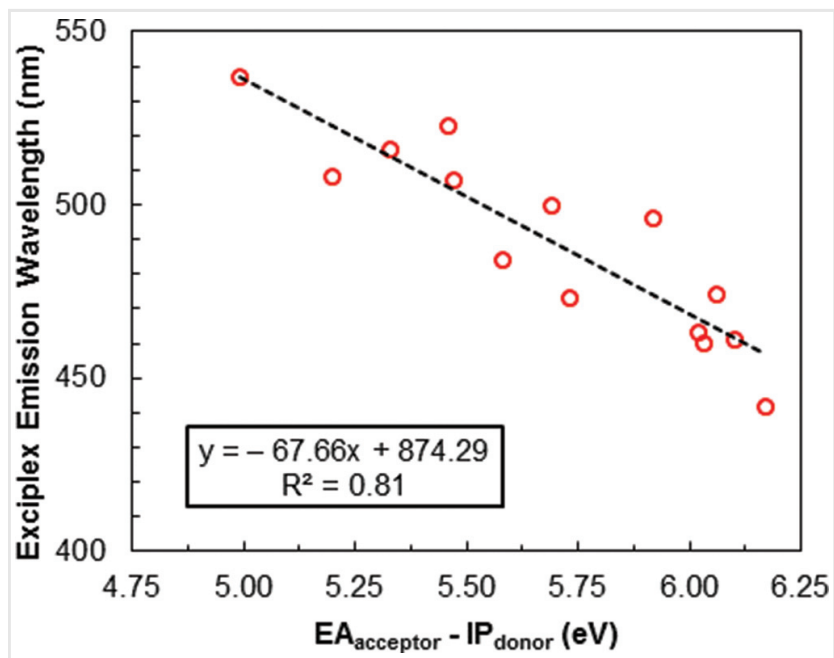

Figure 9 Empirical relationship between experimental exciplex emission wavelength of acceptor 1 and triarylamine donors X01-B11 and the calculated difference of electron affinity (EA) and ionization potential (IP). exciplexemission wavelength also depends on the geometrical distortions in the excited complex, ${ }^{66}$ such relationships have to be established separately for every donor family.

\section{Delayed Fluorescence Measurements}

Owing to its bright emission and preferable emission wavelength, we focus on the exciplex with donor B01. Figure 10 shows the transient PL for the 1:1 mixture of donor B01 and acceptor $\mathbf{1}$ in a PMMA matrix. While exhibiting biexponential delayed fluorescence with lifetimes of $\tau=4.68$ and $45.1 \mu \mathrm{s}$, we further confirm the existence of TADF by performing temperature-dependent transient PL measurement. Upon cooling the sample down to $150 \mathrm{~K}$, the relative contribution from delayed fluorescence drops by approximately a factor of 2 (Supporting Information), indicating that the delayed fluorescence at room temperature is thermally activated. Lifetimes of further exciplexes can be found in the Supporting Information but were generally similar.

\section{OLED Device Studies}

In order to check the feasibility for acceptor $\mathbf{1}+$ donor B01 exciplex in OLED device applications, we fabricated the test device with the structure of: indium tin oxide (ITO, $132 \mathrm{~nm}) /$ di-[4[(N,N-ditolyl-amino)-phenyl]-cyclohexane (TAPC, 150nm)/acceptor 1:donor B01 approx. 1:1 molar ratio $(15 \mathrm{~nm}) / 1,3,5$-tris(3-pyridyl-3-phenyl)benzene (TmPyPB, $60 \mathrm{~nm}) / \mathrm{LiF}(1 \mathrm{~nm}) / \mathrm{Al}(100 \mathrm{~nm})$. ITO and $\mathrm{Al}$ are the anode and cathode, respectively. TAPC serves as the hole-transport layer (HTL) and TmPyPB is the electron-transport layer (ETL).

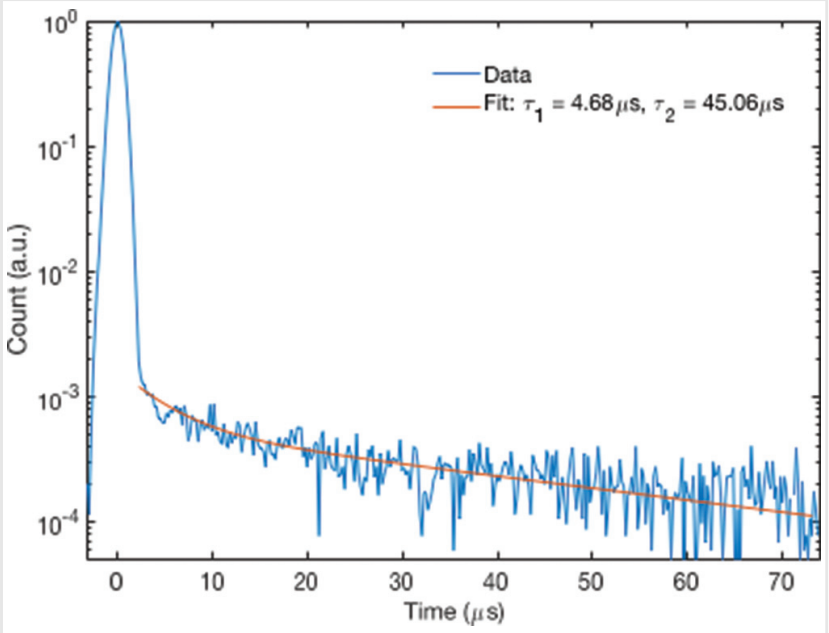

Figure 10 Transient of delayed fluorescence from thin film of acceptor 1 and B01 (1:1 molar ratio) in PMMA. 

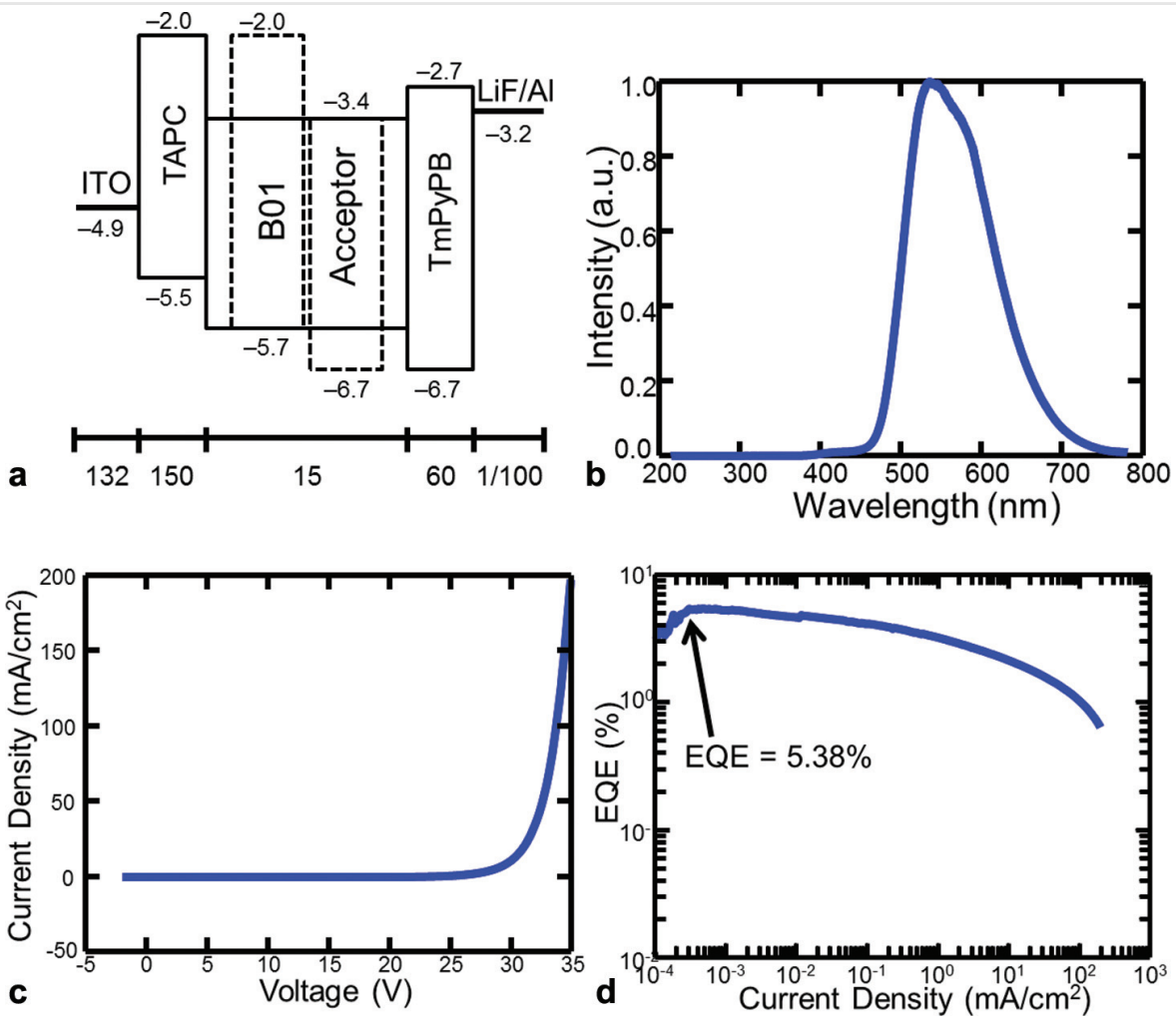

Figure 11 (a) Device structure and the energy band diagrams for the OLED device. The energy levels are labeled in electron volts and the thicknesses are in nanometers. (b) Electroluminescence spectra of the device. (c) The device IV curve. (d) External quantum efficiencies (EQEs) with different current densities.

In addition, we inserted LiF between the ETL and the cathode to boost electron injection (Figure 11a).

The electroluminescence (EL) spectrum of a device is shown in Figure 11b. Intriguingly, instead of showing blue emission as expected from the PL (461 nm, Figure 6), the EL is red-shifted by $75 \mathrm{~nm}$, having its emission peak at $536 \mathrm{~nm}$. The red-shift is too large to attribute to the optical cavity in the device. Transient PL measurements, however, showed that the delayed fluorescence is red-shifted relative to the prompt luminescence (Supporting Information). The shift is consistent with solid-state solvation ${ }^{69-71}$ as the molecules surrounding an emissive state polarize in response to the excited-state dipole. The effect is enhanced in the OLED relative to PL because EL is dominated by carrier condensation to give long-lived triplet excited states rather than photoexcited singlet states.

Figure 11c,d shows the device characteristics. The large turn on voltage may originate from the lack of a host material to facilitate charge transport in the emitting layer. From Figure 11d, a maximum EQE of 5.4\% is achieved, which is comparable to those of other exciplex OLEDs. ${ }^{38}$ Optimization of the device structure, such as introducing a suitable host material for better charge transport and balance, or tuning thickness for each layer to enhance out-coupling efficiency, can be done to further improve the device overall performance.

\section{Conclusions}

In summary, we have designed a supramolecular system featuring a lock-and-key concept, guided by density functional theory calculations. The $C_{3}$-symmetric nature of the acceptor (lock) allows strong binding with three complementarily shaped donor families (keys). Lock-and-keys are demonstrated to be exciplexes, with emission colors covering a large portion of the visible spectrum. Empirical relationships between experimental exciplex wavelengths are established and can be used for color tuning. The exciplex with acceptor $\mathbf{1}$ and $\mathbf{B 0 1}$ displays TADF, which is supported by transients of the delayed fluorescence as well as temperaturedependent studies. EQEs of up to 5.4\% are achieved in an OLED.

\section{Experimental Section}

Commercial chemicals were used without further purification. NMR spectra were recorded on a Bruker 
Avance $400 \mathrm{MHz}$ spectrometer. The residual solvent peaks were used as internal standards. ${ }^{72}$ Mass spectra were measured with a Bruker Daltonics APEX IV 4.7 Tesla Fourier Transform Ion Cyclotron Resonance Mass Spectrometer in electrospray ionization (ESI) or direct analysis in real time (DART) mode. Infrared (IR) spectra were measured on a Thermo Scientific Nicolet 6700 Fourier Transform Infrared (FT-IR) spectrometer in attenuated total reflection (ATR) mode on a germanium crystal. Atmospheric suppression correction was applied. Thin-films of single components were spin coated (4000 rpm, $30 \mathrm{~s}$ ) from $10 \mathrm{mM}$ solutions in chloroform; mixtures from $5 \mathrm{mM}$ solutions respectively. Samples with PMMA matrix contained $5 \mathrm{wt} \%$ of the single component. The films were spin coated on micro cover glasses by VWR.

Solution UV/VIS absorption spectra were obtained with an Agilent Cary 4000 UV/VIS spectrophotometer. Thin-film UV/VIS absorption spectra were obtained with an Agilent 8453 UV/VIS spectroscopy system. Photoluminescence spectra were measured with a Horiba Jobin Yvon Fluorolog-3 spectrofluorometer (model FL3-21) employing a $450 \mathrm{~W}$ xenon short-arc lamp. Excitation and emission bandpass slits were set at $3 \mathrm{~nm}$ and $5 \mathrm{~nm}$, respectively. Donor molecules were excited at their absorption maxima; acceptor 1 at $336 \mathrm{~nm}$ respectively. For 1:1 mixtures of donors with acceptor 1 an excitation wavelength of 336 or $356 \mathrm{~nm}$ was used.

The thin-film for the delayed fluorescence measurements was fabricated through drop-casting, with $37 \mathrm{wt} \%$ of acceptor $\mathbf{1}+\mathbf{B 0 1}$ in PMMA. Acceptor to B01 ratio is 1:1 in molar ratio. The transient $\mathrm{PL}$ was measured with a Hamamatsu streak camera at $375 \mathrm{~nm}$ excitation, with a repetition rate of $220 \mathrm{~Hz}$ to ensure full decay of the delayed fluorescence. Temperature-dependent transient PL was performed by cooling down the sample to $150 \mathrm{~K}$ with a Helium-cooled cryostat, using the same setup and measurement parameters as room temperature measurement. Transient photoluminescence lifetime measurements of all other exciplexes were performed with a $337 \mathrm{~nm} \mathrm{~N} \mathrm{~N}_{2}$ laser (NL 100, Stanford Research Systems) as the excitation source. The photoluminescence was detected with a Thorlabs PDA10A Si detector. The resulting time-dependent photoluminescence data were recorded using a Tektronix TDS3054C oscilloscope.

Electrochemical measurements were carried out in a glove box under nitrogen, using an Autolab PGSTAT 10 or PGSTAT 20 potentiostat (Eco Chemie) in a three-electrode cell configuration. A Pt button (1.6 mm in diameter) electrode, a Pt wire, and a quasi-internal $\mathrm{Ag}$ wire submerged in $0.01 \mathrm{M} \mathrm{AgNO}_{3} / 0.1 \mathrm{M}$ tetrabutylammonium hexafluorophosphate $\left(\mathrm{TBAPF}_{6}\right)$ in acetonitrile were used as a working electrode, a counter electrode, and a reference electrode, respectively, in $0.1 \mathrm{M} \mathrm{TBAPF}{ }_{6}$ dichloromethane solution. The ferrocene/ferrocenium $\left(\mathrm{Fc} / \mathrm{Fc}^{+}\right)$redox couple was used as an internal standard, with the half-wave potentials observed between $0.195-0.205 \mathrm{~V}$ vs $\mathrm{Ag} / \mathrm{Ag}^{+}$in dichloromethane solution.

\section{Device Fabrication and Characterization}

The testing OLED devices were made on a pre-cleaned and pre-patterened indium tin oxide (ITO) on glass substrate. The ITO is $132 \mathrm{~nm}$ thick. All the other OLED layers in the device were deposited subsequently by thermal evaporation in a vacuum chamber with base pressure of $<10^{-6}$ torr. These layers include: $\mathrm{Di}-[4[(\mathrm{~N}, \mathrm{~N}-$ ditolyl-amino)-phenyl]-cyclohexane (TAPC, $150 \mathrm{~nm}$ )/ emitting materials layer (acceptor 1:donor B01 $=1: 1$ in molar ratio, $15 \mathrm{~nm}$ ) / 1,3,5-Tris(3-pyridyl-3-phenyl)benzene (TmPyPB, $60 \mathrm{~nm}) / \mathrm{LiF}(1 \mathrm{~nm}) / \mathrm{Al}(100 \mathrm{~nm})$. The EML was fabricated by co-evaporation from two separate evaporation sources at the same rate to achieve 1:1 volume ratio for acceptor $\mathbf{1}$ and donor B01. The devices are then packaged under nitrogen glovebox with UV-cured epoxy to maintain inert environment during measurements. The device currents and photocurrents are then measured with a 4156C Precision Semiconductor Parameter Analyzer. The electroluminescence spectra of the devices are measured with a Princeton Instruments SP2300 and PIXIS-100 spectrometer.

Density functional theory calculations were performed with the QChem 4.3 software package ${ }^{73}$ using the B3LYP functional $^{74}$ with Grimme's dispersion corrections. ${ }^{75} \mathrm{~A}$ Lebedev $(75,302)$ exchange-correlation grid $^{76}$ was used. Geometry optimizations were performed using a $6-31+\mathrm{G}^{*}$ basis set. Binding energies, HOMOs, and IPs were computed in an aug-cc-pVDZ basis set. ${ }^{77}$ Ionization potentials were computed by vertical detachment of an electron. Electron affinities were computed by vertical attachment of an electron.

\section{Funding Information}

This study was funded by the Air Force Office of Scientific Research (FA9550-18-1-0341), the Department of Energy, Labor and Economic Growth (DE-FG02-07ER46474), DFG (EN 1138/1-1), NIH (GM112272), and Schweizerischer Nationalfonds zur Förderung der Wissenschaftlichen Forschung (PA00P2-145389).

\section{Acknowledgment}

This research was supported in part by the Air Force Office of Scientific Research (FA9550-18-1-0341 17RT0904) and Samsung Corp. We thank Dr. Hyun Sik Chae for discussion. 
G.M. thanks the German Academic Exchange Service (DAAD) for a postdoctoral fellowship. J.U.E. thanks the German research foundation (DFG, Project EN 1138/1-1) for financial support. S.R. was supported through an advanced postdoctoral fellowship from the Swiss National Science Foundation (PA00P2-145389). G.T.S. was supported by a F32 Ruth L. Kirschstein NRSA fellowship under award number GM112272. T.C.W. and T.L. were supported by the US Department of Energy, Office of Basic Energy Sciences (Award No. DE-FG02-07ER46474). The authors acknowledge help from Dong-Gwang Ha for transient PL measurement.

\section{Supporting Information}

Supporting information for this article is available online at https://doi.org/10.1055/s-0039-3402059.

\section{References}

(1) Endo, A.; Sato, K.; Yoshimura, K.; Kai, T.; Kawada, A.; Miyazaki, H.; Adachi, C. Appl. Phys. Lett. 2011, 98, 083302.

(2) Uoyama, H.; Goushi, K.; Shizu, K.; Nomura, H.; Adachi, C. Nature 2012, 492, 234.

(3) Zhang, Q.; Li, B.; Huang, S.; Nomura, H.; Tanaka, H.; Adachi, C. Nat. Photonics 2014, 8, 326.

(4) Tao, Y.; Yuan, K.; Chen, T.; Xu, P.; Li, H.; Chen, R.; Zheng, C.; Zhang, L.; Huang, W. Adv. Mater. 2014, 26, 7931.

(5) Volz, D.; Wallesch, M.; Fléchon, C.; Danz, M.; Verma, A.; Navarro, J. M.; Zink, D. M.; Bräse, S.; Baumann, T. Green Chem. 2015, 17, 1988.

(6) Cho, Y. J.; Yook, K. S.; Lee, J. Y. Adv. Mater. 2014, 26, 4050.

(7) Lee, D. R.; Kim, B. S.; Lee, C. W.; Im, Y.; Yook, K. S.; Hwang, S. H.; Lee, J. Y. ACS Appl. Mater. Inter. 2015, 7, 9625.

(8) Sun, J. W.; Lee, J. H.; Moon, C. K.; Kim, K. H.; Shin, H.; Kim, J. J. Adv. Mater. 2014, 26, 5684.

(9) Wu, T. L.; Huang, M. J.; Lin, C. C.; Huang, P. Y.; Chou, T. Y.; ChenCheng, R. W.; Lin, H. W.; Liu, R. S.; Cheng, C. H. Nat. Photonics 2018, 12, 235.

(10) Liu, Y.; Li, C.; Ren, Z.; Yan, S.; Bryce, M. R. Nat. Rev. Mater. 2018, 3 , 18020.

(11) Méhes, G.; Nomura, H.; Zhang, Q.; Nakagawa, T.; Adachi, C. Angew. Chem. Int. Ed. Engl. 2012, 51, 11311.

(12) Kawasumi, K.; Wu, T.; Zhu, T.; Chae, H. S.; Van Voorhis, T.; Baldo, M. A.; Swager, T. M. J. Am. Chem. Soc. 2015, 137, 11908.

(13) Voll, C.-C. A.; Engelhart, J.; Einzinger, M.; Baldo, M. A.; Swager, T. M. Eur. J. Org. Chem. 2017, 4846.

(14) Goushi, K.; Adachi, C. Appl. Phys. Lett. 2012, 101, 023306.

(15) Goushi, K.; Yoshida, K.; Sato, K.; Adachi, C. Nat. Photonics 2012, 6, 253.

(16) Chen, Z.; Liu, X. K.; Zheng, C. J.; Ye, J.; Liu, C. L.; Li, F.; Ou, X. M.; Lee, C. S.; Zhang, X. H. Chem. Mater. 2015, 27, 5206.

(17) Oh, C. S.; Kang, Y. J.; Jeon, S. K.; Lee, J. Y. J. Phys. Chem. C. 2015, 119, 22618.

(18) Zhang, L.; Cai, C.; Li, K. F.; Tam, H. L.; Chan, K. L.; Cheah, K. W. ACS Appl. Mater. Inter. 2015, 7, 24983.

(19) Zhao, B.; Zhang, T.; Chu, B.; Li, W.; Su, Z.; Luo, Y.; Li, R.; Yan, X.; Jin, F.; Gao, Y.; Wu, H. Org. Electron. 2015, 17, 15.
(20) Zhang, T.; Zhao, B.; Chu, B.; Li, W.; Su, Z.; Wang, L.; Wang, J.; Jin, F.; Yan, X.; Gao, Y.; Wu, H.; Liu, C.; Lin, T.; Hou, F. Org. Electron. 2015, 24, 1 .

(21) Zhang, T.; Zhao, B.; Chu, B.; Li, W.; Su, Z.; Yan, X.; Liu, C.; Wu, H.; Jin, F.; Gao, Y. Org. Electron. 2015, 25, 6.

(22) Chen, D.; Wang, Z.; Wang, D.; Wu, Y. C.; Lo, C. C.; Lien, A.; Cao, Y.; Su, S. J. Org. Electron. 2015, 25, 79.

(23) Chen, D.; Xie, G.; Cai, X.; Liu, M.; Cao, Y.; Su, S. J. Adv. Mater. 2016 $28,239$.

(24) Liu, W.; Chen, J.-X.; Zheng, C.-J.; Wang, K.; Chen, D.-Y.; Li, F.; Dong, Y.-P.; Lee, C.-S.; Ou, X.-M.; Zhang, X.-H. Adv. Funct. Mater. 2016, 26, 2002.

(25) Kim, K. H.; Yoo, S. J.; Kim, J. J. Chem. Mater. 2016, 28, 1936.

(26) Li, J.; Nomura, H.; Miyazaki, H.; Adachi, C. Chem. Commun. (Camb.) 2014, 50, 6174.

(27) Hung, W. Y.; Chiang, P. Y.; Lin, S. W.; Tang, W. C.; Chen, Y. T.; Liu, S. H.; Chou, P. T.; Hung, Y. T.; Wong, K. T. ACS Appl. Mater. Inter. 2016, 8, 4811.

(28) Jeon, S. K.; Yook, K. S.; Lee, J. Y. Nanotechnology 2016, 27, 224001.

(29) Jankus, V.; Chiang, C. J.; Dias, F.; Monkman, A. P. Adv. Mater. 2013, $25,1455$.

(30) Data, P.; Kurowska, A.; Pluczyk, S.; Zassowski, P.; Pander, P.; Jedrysiak, R.; Czwartosz, M.; Otulakowski, L.; Suwinski, J.; Lapkowski, M.; Monkman, A. P. J. Phys. Chem. C. 2016, 120, 2070.

(31) Park, Y. S.; Kim, K. H.; Kim, J. J. Appl. Phys. Lett. 2013, 102, 153306.

(32) Hung, W. Y.; Fang, G. C.; Chang, Y. C.; Kuo, T.; Chou, P.; Lin, S.; Wong, K. ACS Appl. Mater. Inter. 2013, 5, 6826.

(33) Huang, Q.; Zhao, S.; Xu, Z.; Fan, X.; Shen, C.; Yang, Q. Appl. Phys. Lett. 2014, 104, 161112.

(34) Hung, W. Y.; Fang, G. C.; Lin, S. W.; Cheng, S. H.; Wong, K. T.; Kuo, T. Y.; Chou, P. T. Sci. Rep. 2014, 4, 5161.

(35) Zhang, T.; Chu, B.; Li, W.; Su, Z.; Peng, Q. M.; Zhao, B.; Luo, Y.; Jin, F.; Yan, X.; Gao, Y.; Wu, H.; Zhang, F.; Fan, D.; Wang, J. ACS Appl. Mater. Inter. 2014, 6, 11907.

(36) Liu, X. K.; Chen, Z.; Zheng, C. J.; Liu, C. L.; Lee, C. S.; Li, F.; Ou, X. M.; Zhang, X. H. Adv. Mater. 2015, 27, 2378.

(37) Liu, X. K.; Chen, Z.; Qing, J.; Zhang, W. J.; Wu, B.; Tam, H. L.; Zhu, F.; Zhang, X. H.; Lee, C. S. Adv. Mater. 2015, 27, 7079.

(38) Sarma, M.; Wong, K. T. ACS Appl. Mater. Inter. 2018, 10, 19279.

(39) Chapran, M.; Pander, P.; Vasylieva, M.; Wiosna-Sałyga, G.; Ulanski, J.; Dias, F. B.; Data, P. ACS Appl. Mater. Inter. 2019, 11, 13460.

(40) Hung, Y. T.; Chen, Z. Y.; Hung, W. Y.; Chen, D. G.; Wong, K. T. ACS Appl. Mater. Inter. 2018, 10, 34435.

(41) Lai, S. L.; Chan, M. Y.; Tong, Q. X.; Fung, M. K.; Wang, P. F.; Lee, C. S.; Lee, S. T. Appl. Phys. Lett. 2008, 93, 143301.

(42) Fischer, E. Ber. Dtsch. Chem. Ges. 1894, 27, 2985.

(43) Behr, J. P., ed. The Lock-and-Key Principle. The State of the Art 100 Years on. John Wiley \& Sons Ltd: Chichester, 1994.

(44) Chiba, T.; Pu, Y. J.; Miyazaki, R.; Nakayama, K. I.; Sasabe, H.; Kido, J. Org. Electron. 2011, 12, 710.

(45) Cho, S. H.; Pyo, S. W.; Suh, M. C. Synth. Met. 2012, 162, 402.

(46) Kim, Y. K.; Won Kim, J.; Park, Y. Appl. Phys. Lett. 2009, 94, 63305.

(47) Lee, S.; Lee, J. H.; Lee, J. H.; Kim, J. J. Adv. Funct. Mater. 2012, 22, 855.

(48) Liao, L. S.; Klubek, K. P. Appl. Phys. Lett. 2008, 92, 223311.

(49) Liao, L. S.; Slusarek, W. K.; Hatwar, T. K.; Ricks, M. L.; Comfort, D. L. Adv. Mater. 2008, 20, 324.

(50) Lin, H. W.; Lin, W. C.; Chang, J. H.; Wu, C. I. Org. Electron. 2013, 14, 1204.

(51) Park, S. M.; Kim, Y. H.; Yi, Y.; Oh, H. Y.; Won Kim, J. Appl. Phys. Lett. 2010, 97, 063308. 
(52) Small, C. E.; Tsang, S. W.; Kido, J.; So, S. K.; So, F. Adv. Funct. Mater. 2012, 22, 3261.

(53) Yook, K. S.; Jeon, S. O.; Lee, J. Y. Thin Solid Films 2009, 517, 6109.

(54) Swager, T. M. Acc. Chem. Res. 2008, 41, 1181.

(55) Mahmood, J.; Kim, D.; Jeon, I. Y.; Lah, M. S.; Baek, J. B. Synlett. 2013, 24, 246.

(56) Wright, M. W.; Welker, M. E. J. Org. Chem. 1996, 61, 133.

(57) Tan-Sien-Hee, L.; Mesmaeker, A. K.-D. J. Chem. Soc. Dalt. Trans. 1994, 3651.

(58) Juárez, R.; Moreno Oliva, M.; Ramos, M.; Segura, J. L.; Alemán, C.; Rodríguez-Ropero, F.; Curcó, D.; Montilla, F.; Coropceanu, V.; Brédas, J. L.; Qi, Y.; Kahn, A.; Ruiz Delgado, M. C.; Casado, J.; López Navarrete, J. T. Chem. Eur. J. 2011, 17, 10312.

(59) Harada, N.; Uda, H.; Nakasuji, K.; Murata, I. J. Chem. Soc., Perkin Trans. 2 1989, 1449.

(60) Cardona, C. M.; Li, W.; Kaifer, A. E.; Stockdale, D.; Bazan, G. C. Adv. Mater. 2011, 23, 2367.

(61) Kuwabara, Y.; Ogawa, H.; Inada, H.; Noma, N.; Shirota, Y. Adv. Mater. 1994, 6, 677.

(62) Surry, D. S.; Buchwald, S. L. J. Am. Chem. Soc. 2007, 129, 10354.

(63) Parac, M.; Etinski, M.; Peric, M.; Grimme, S. J. Chem. Theory Comput. 2005, 1, 1110.

(64) Kashiki, T.; Shinamura, S.; Kohara, M.; Miyazaki, E.; Takimiya, K.; Ikeda, M.; Kuwabara, H. Org. Lett. 2009, 11, 2473.

(65) Beens, H.; Weller, A. Excited Molecular $\pi$-Complexes in Solution. In: Organic Molecular Photophysics, Vol 2. Birks, J. B., ed.; John Wiley \& Sons: London, New York, 1973, 159.

(66) Förster, T. Excimers and Exciplexes. In: The Exciplex. Ware, W. R.; Gordon, M., eds.; Academic Press: New York, 1974, $1-22$.

(67) A plot of the exciplex emission energy against the calculated EAIP difference is included in the Supporting Information.
(68) As a computationally convenient alternative we show in the Supporting Information that the HOMO and LUMO energies from DFT calculations can also be used for a sufficiently good approximation. In fact, the $R^{2}=0.85$ is even better, which might be a result of fortuitous error cancellation.

(69) Méhes, G.; Goushi, K.; Potscavage, W. J.; Adachi, C. Org. Electron. 2014, 15, 2027.

(70) Madigan, C. F.; Bulović, V. Phys. Rev. Lett. 2003, 91, 247403.

(71) Baldo, M. A.; Soos, Z. G.; Forrest, S. R. Chem. Phys. Lett. 2001, 347, 297.

(72) Gottlieb, H. E.; Kotlyar, V.; Nudelman, A. J. Org. Chem. 1997, 62, 7512.

(73) Shao, Y.; Molnar, L. F.; Jung, Y.; Kussmann, J.; Ochsenfeld, C.; Brown, S. T.; Gilbert, A. T.B.; Slipchenko, L. V.; Levchenko, S. V.; O’Neill, D. P.; DiStasio, R. a; Lochan, R. C.; Wang, T.; Beran, G. J.O.; Besley, N. a; Herbert, J. M.; Lin, C. Y.; Van Voorhis, T.; Chien, S. H.; Sodt, A.; Steele, R. P.; Rassolov, V. a; Maslen, P. E.; Korambath, P. P.; Adamson, R. D.; Austin, B.; Baker, J.; Byrd, E. F. C.; Dachsel, H.; Doerksen, R. J.; Dreuw, A.; Dunietz, B. D.; Dutoi, A. D.; Furlani, T. R.; Gwaltney, S. R.; Heyden, A.; Hirata, S.; Hsu, C.-P.; Kedziora, G.; Khalliulin, R. Z.; Klunzinger, P.; Lee, A. M.; Lee, M. S.; Liang, W.; Lotan, I.; Nair, N.; Peters, B.; Proynov, E. I.; Pieniazek, P. a; Rhee, Y. M.; Ritchie, J.; Rosta, E.; Sherrill, C. D.; Simmonett, A. C.; Subotnik, J. E.; Woodcock, H. L.; Zhang, W.; Bell, A. T.; Chakraborty, A. K.; Chipman, D. M.; Keil, F. J.; Warshel, A.; Hehre, W. J.; Schaefer, H. F.; Kong, J.; Krylov, A. I.; Gill, P. M. W.; Head-Gordon, M. Phys. Chem. Chem. Phys. 2006, 8, 3172.

(74) Becke, A. D. J. Chem. Phys. 1993, 98, 1372.

(75) Grimme, S.; Antony, J.; Ehrlich, S.; Krieg, H. J. Chem. Phys. 2010, 132, 154104.

(76) Lebedev, V. I. Sib. Math. J. 1977, 18, 132.

(77) Krishnan, R.; Binkley, J. S.; Seeger, R.; Pople, J. A.; Krishnan, R.; Binkley, J. S.; Seeger, R.; Pople, J. A. J. Chem. Phys. 1980, 72, 650. 